\title{
Japanese Expert Panel Meeting on the Management of Prostate Cancer with Bone Metastases
}

\author{
Shunji Takahashi · Seigo Kinuya · Norio Nonomura · Nobuo Shinohara $\cdot$ Kazuhiro Suzuki · Hiroyoshi Suzuki • \\ Katsumasa Nakamura · Takefumi Satoh • Ukihide Tateishi · Toshiyuki Yoneda · Hiroyuki Horikoshi • \\ Tsukasa Igawa · Takao Kamai · Mitsuru Koizumi · Takeo Kosaka • Nobuaki Matsubara · Hideaki Miyake • \\ Atsushi Mizokami · Takashi Mizowaki · Naoki Nakamura · Masahiro Nozawa · Takeo Takahashi • \\ Hiroji Uemura $\cdot$ Motohide Uemura $\cdot$ Akira Yokomizo $\cdot$ Mana Yoshimura $\cdot$ Yoshiyuki Kakehi
}

Received: September 11, 2018 / Published online: December 11, 2018

(C) The Author(s) 2018

\section{ABSTRACT}

Introduction: The incidence of prostate cancer in Japan continues to increase, necessitating the continued development of effective therapies and strategies. Recent advances in treatments have improved the prognosis of metastatic disease and highlighted the importance of treating bone metastases to reduce the incidence of skeletal complications and improve patients' quality of life. With the increasing number of treatment options that have become available, including bone-targeted therapy with the alpha

Enhanced digital features To view enhanced digital features for this article go to https://doi.org/10.6084/ m9.figshare.7306703.

Electronic supplementary material The online version of this article (https://doi.org/10.1007/s40487018-0088-0) contains supplementary material, which is available to authorized users.

S. Takahashi $(\bowtie) \cdot$ M. Koizumi

The Cancer Institute Hospital of the Japanese

Foundation for Cancer Research, Koto-ku, Tokyo, Japan

e-mail: s.takahashi-chemotherapy@jfcr.or.jp

S. Kinuya - A. Mizokami

Kanazawa University, Kanazawa, Ishikawa, Japan

N. Nonomura $\cdot$ M. Uemura

Osaka University, Yamadaoka, Suita, Osaka, Japan

N. Shinohara

Hokkaido University, Sapporo, Hokkaido, Japan emitter radium-223 dichloride (Ra-223), Japanese clinicians are faced with making difficult decisions on the choice of optimal treatment strategy. In such situations, guidance based on expert opinions can be beneficial.

Methods: A panel meeting of 27 Japanese experts in the management of prostate cancer was held to share opinions and to establish consensus recommendations on key clinical questions. Panelists were asked to vote on more than 40 questions pertinent to prostate cancer, and the answers helped guide a comprehensive discussion.

Results: The panel reached a consensus on key topics related to the optimal treatment strategy for Ra-223 therapy, namely, that patients with symptomatic, metastatic castration-resistant prostate cancer (CRPC) would benefit most from the use of this agent and that this treatment therapy should be provided before chemotherapy. Other topics that achieved

K. Suzuki

Gunma University, Maebashi, Gunma, Japan

H. Suzuki

Toho University Sakura Medical Center, Sakura,

Chiba, Japan

K. Nakamura · H. Miyake

Hamamatsu University School of Medicine,

Hamamatsu, Shizuoka, Japan

T. Satoh

Kitasato University School of Medicine, Sagamihara, Kanagawa, Japan 
consensus included: monitoring for osteoporosis and providing treatment if necessary during androgen deprivation therapy; performing magnetic resonance imaging in the presence of discrepancies in bone scintigram and computed tomography scans; monitoring alkaline phosphatase during CRPC treatment; using osteoclast-targeting in patients with CRPC with bone metastases; and using osteoclast-targeted agents combined with Ra-223.

Conclusion: These consensus recommendations and the updated information which became available subsequent to the panel meeting included here provide useful information for clinicians to aid in designing optimal treatment strategies for their patients.

Funding: Bayer Yakuhin Ltd.

Keywords: Bone metastasis; Castrationresistant; Japanese; Prostate cancer; Therapeutic consensus

\section{INTRODUCTION}

The incidence of prostate cancer is increasing among Japanese males, with an estimated 86,100 new cases diagnosed by the National

T. Satoh

Sato Takefumi Zenritsusen Clinic, Machida, Tokyo, Japan

U. Tateishi

Tokyo Medical and Dental University, Bunkyo-ku,

Tokyo, Japan

T. Yoneda

Osaka University Graduate School of Dentistry,

Suita, Osaka, Japan

T. Yoneda

Indiana University, Bloomington, IN, USA

H. Horikoshi

Gunma Prefectural Cancer Center, Ohta, Gunma, Japan

T. Igawa

Kurume University, Fukuoka, Fukuoka, Japan

T. Kamai

Dokkyo Medical University, Shimotsuga-gun,

Tochigi, Japan
Cancer Center Japan in 2017 [1]. For patients with metastatic disease, recent advances in treatment strategies have improved their prognosis and disease management. As the most frequent metastatic site of prostate cancer is bone, it is important to treat bone metastases in order to reduce the incidence of skeletal complications and improve patients' quality of life.

In Japan, various therapeutic options are now available for the treatment of metastatic prostate cancer. As a result, clinicians are often faced with the daunting challenge of determining the optimal management strategy for an individual patient, including diagnosis and monitoring. To facilitate the decision-making process regarding the optimal treatment for a patient who has castration-resistant prostate cancer (CRPC) with bone metastases, a thorough review of the recent advances in basic and clinical research, which have resulted in the introduction of drugs with novel modalities, is required .

For decades, prostate cancer was treated primarily with androgen deprivation therapy (ADT). Docetaxel (DOC) was introduced at the beginning of the twenty-first century and has proven to be effective in the treatment of CRPC. In 2014, enzalutamide (ENZ) and abiraterone $(\mathrm{ABI})$ were introduced in Japan as treatments for CRPC, as was cabazitaxel, after trials reported

T. Kosaka

Keio University, 35 Shinanomachi, Shinjuku-ku, Tokyo, Japan

N. Matsubara $\cdot$ N. Nakamura

National Cancer Center Hospital East, Kashiwa, Chiba, Japan

T. Mizowaki

Kyoto University, Sakyo, Kyoto, Japan

M. Nozawa

Faculty of Medicine, Kindai University, Osaka-

Sayama, Osaka, Japan

T. Takahashi

Saitama Medical University, Kawagoe, Saitama, Japan

H. Uemura

Yokohama City University Medical Center, Yokohama, Kanagawa, Japan

A. Yokomizo

Harasanshin Hospital, Fukuoka, Fukuoka, Japan 
improvements in survival following treatment with DOC. Bone-targeted therapy with the alpha emitter radium-223 dichloride (Ra-223) was approved in Japan in June 2016. Ra-223 is the first agent found to improve both overall survival (OS) and time to symptomatic skeletal events, as shown in the ALSYMPCA trial $[2,3]$; however, optimal patient selection and the optimal timing to initiate therapy have yet to be determined. Given the currently limited realworld experience with Ra-223, expert opinions, rather than clinical guidelines offering evidence-based recommendations, would be helpful for such decision-making in daily clinical practice.

We therefore organized a Japanese expert panel meeting for the management of prostate cancer with bone metastases, a forum at which experts could share their experiences and reach consensus on the key clinical questions related to the management of prostate cancer with bone metastases. The main focus of this meeting was to determine the optimal incorporation of Ra-223 into CRPC therapeutic regimens, but panelists also discussed other important and controversial topics in the areas of imaging and monitoring of prostate cancer.

\section{METHODS}

Held on September 3, 2016, in Tokyo, Japan, the consensus panel included 27 experts from a variety of specialties involved in the management of prostate cancer (Table 1). Of the panel participants, 96\% were actively working at institutions that met facility requirements for the use of Ra-223, although $60 \%$ did not have any personal experience with Ra-223 treatment in clinical practice (except in clinical trials) at the time of meeting. Steering committee

\footnotetext{
A. Yokomizo

Kyushu University, Fukuoka, Fukuoka, Japan

M. Yoshimura

Tokyo Medical University, Shinjuku-ku, Tokyo, Japan

Y. Kakehi

Kagawa University, Kita-gun, Kagawa, Japan
}

members were selected from the leadership of societies associated to the field and nominated by the participating panelists with no influence from the sponsor/funding agency.

Since the goal of the panel meeting was to address practical clinical needs, identifying realworld clinical questions was essential. The format of this meeting broadly followed that of the St Gallen Advanced Prostate Cancer Consensus Conference (APCCC) 2015 [4], and the simplified method of the Delphi process was used throughout [5]. Before the meeting, members of the steering committee discussed and agreed upon the goal of the meeting. They identified clinical questions that would address issues pertinent to the management and monitoring of patients with CRPC with bone metastases. panelists were selected for their relevant expertise and were asked to submit examples of clinically relevant questions that are commonly raised during their daily practice. Starting with the list of clinical questions in the current Japanese Urological Association Guidelines, panelists deleted questions that were not clinically relevant to the topic on hand and added others they expected to be asked. The questions gathered using this process were categorized and prioritized by the steering committee, and the final version of the list was presented at the consensus panel meeting.

During the forum, all of the questions were presented to the panel in a multiple-choice format. panelists were polled anonymously using an electronic voting system. Those who were unable to vote for a best choice for any reason could choose 'abstain' as an option (see Electronic Supplementary Material [ESM] Table S1 for details). Each question was voted on only once, and there was no option to revote. After voting, the panel discussed the results and shared opinions.

The data presented in this consensus document are based on the voting results and related discussions and, therefore, do not report on research conducted on human subjects. This article does not contain any studies with human participants or animals performed by any of the authors. All panelists have reviewed and approved this manuscript. 
Table 1 Panel members

\begin{tabular}{|c|c|c|c|}
\hline Role & Name & Specialty & Affiliations \\
\hline $\begin{array}{l}\text { Meeting Chair } \\
\text { SC Member }\end{array}$ & Takahashi, Shunji $^{\mathrm{a}}$ & Medical Oncology & $\begin{array}{l}\text { The Cancer Institute Hospital of the } \\
\text { Japanese Foundation for Cancer Research }\end{array}$ \\
\hline $\begin{array}{l}\text { Meeting Chair } \\
\text { SC Member }\end{array}$ & Kinuya, Seigo & Nuclear Medicine & Kanazawa University \\
\hline $\begin{array}{l}\text { Meeting Chair } \\
\text { SC Member }\end{array}$ & Nonomura, Norio & Urology & Osaka University \\
\hline $\begin{array}{l}\text { Meeting Chair } \\
\text { SC Member }\end{array}$ & Shinohara, Nobuo & Urology & Hokkaido University \\
\hline $\begin{array}{l}\text { Meeting Chair } \\
\text { SC Member }\end{array}$ & Suzuki, Kazuhiro & Urology & Gunma University \\
\hline $\begin{array}{l}\text { Meeting Chair } \\
\text { SC Member }\end{array}$ & Suzuki, Hiroyoshi & Urology & Toho University Sakura Medical Center \\
\hline $\begin{array}{l}\text { Meeting Chair } \\
\text { SC Chair }\end{array}$ & Kakehi, Yoshiyuki & Urology & Kagawa University \\
\hline Plenary Speaker & Nakamura, Katsumasa & Radiation Oncology & Hamamatsu University School of Medicine \\
\hline Plenary Speaker & Satoh, Takefumi & Urology & Kitasato University School of Medicine \\
\hline Plenary Speaker & Tateishi, Ukihide & Nuclear Medicine & Tokyo Medical and Dental University \\
\hline Plenary Speaker & Yoneda, Toshiyuki & Basic Science Research & Indiana University ${ }^{\mathrm{b}}$ \\
\hline Panelist & Horikoshi, Hiroyuki & Radiation Diagnostic & Gunma Prefectural Cancer Center \\
\hline Panelist & Igawa, Tsukasa & Urology & Kurume University \\
\hline Panelist & Kamai, Takao & Urology & Dokkyo Medical University \\
\hline Panelist & Koizumi, Mitsuru & Nuclear Medicine & $\begin{array}{l}\text { The Cancer Institute Hospital of the } \\
\text { Japanese Foundation for Cancer Research }\end{array}$ \\
\hline Panelist & Kosaka, Takeo & Urology & Keio University \\
\hline Panelist & Matsubara, Nobuaki & Medical Oncology & National Cancer Center Hospital East \\
\hline Panelist & Miyake, Hideaki & Urology & Hamamatsu University School of Medicine \\
\hline Panelist & Mizokami, Atsushi & Urology & Kanazawa University \\
\hline Panelist & Mizowaki, Takashi & Radiation Oncology & Kyoto University \\
\hline Panelist & Nakamura, Naoki & Radiation Oncology & National Cancer Center Hospital East \\
\hline Panelist & Nozawa, Masahiro & Urology & Kindai University \\
\hline Panelist & Takahashi, Takeo & Radiation Oncology & Saitama Medical University \\
\hline Panelist & Uemura, Hiroji & Urology & Yokohama City University Medical Center \\
\hline Panelist & Uemura, Motohide & Urology & Osaka University \\
\hline
\end{tabular}


Table 1 continued

\begin{tabular}{llll}
\hline Role & Name & Specialty & Affiliations \\
\hline Panelist & Yokomizo, Akira & Urology & Kyushu University \\
Panelist & Yoshimura, Mana & Nuclear Medicine & Tokyo Medical University \\
\hline
\end{tabular}

$S C$ Steering Committee

a First/corresponding author

b Affiliation at the time of meeting

\section{RESULTS}

The voting results on the questions are discussed in detail in the following sub-sections, with the complete dataset shown in the ESM Table S1 and ESM Figs. S1-S30. The total number of voters for each question included those who chose to abstain; however, the numbers vary between questions because some panelists did not vote on all questions.

\section{Management of Castration-Sensitive Prostate Cancer}

First we queried the panelists about their practices in the management of castration-sensitive prostate cancer (CSPC) and the assessment and treatment of bone disease in CSPC.

Combined Androgen Blockade as Primary ADT At the time of this consensus meeting the Japanese Urological Association Guidelines recommended combined androgen blockade $(\mathrm{CAB})$ as the primary ADT (PADT) in patients with M1 prostate cancer [6], and 73\% (19/26) of the panel members agreed with that recommendation. In contrast, half of the panel at the APCCC 2015 did not recommend $\mathrm{CAB}$ as treatment [7].

\section{Treatment Strategy for Patients with Oligometastases}

According to a recent multi-institutional analysis, stereotactic body radiotherapy (SBRT) is associated with a prolonged progression-free survival (PFS) in patients with oligometastatic prostate cancer recurrence who are treatmentnaive [8]. Azzam et al. also reported that prognosis was better for men with recurrent prostate cancer treated with SBRT if they had $\leq 4$ metastases [9]. Regarding the treatment strategy for patients with oligometastases $(\leq 3$ [bone, lymph node]) at initial diagnosis, $38 \%$ of the panelists recommended drug treatment alone, $15 \%$ recommended 'radical therapy' in addition to drug treatment, and 27\% recommended radiation to metastases in addition to drug treatment. Radical therapy may be an option for oligometastases, but it should only be taken after careful evaluation to prevent overlooking micro-metastases.

\section{Osteoclast-Targeting Agents for CSPC}

The panel members felt that regular osteoporosis diagnosis is important; however, as to the timing of initiation of bone-targeted therapies, the opinions of the panel members were divided. For patients with CSPC with bone metastases, $27 \%$ prescribe osteoclast-targeting agents (zoledronic acid or denosumab) to the majority of patients, while $34 \%$ prescribe these agents to a minority of selected patients due to their concern for adverse events, especially osteonecrosis of the jaw (Fig. 1). It should be noted that steoclast-targeting agents were not shown to improve patient prognosis in several randomized trial results available in 2016, such as the STAMPEDE trial [10] and a meta-analysis [11]. 


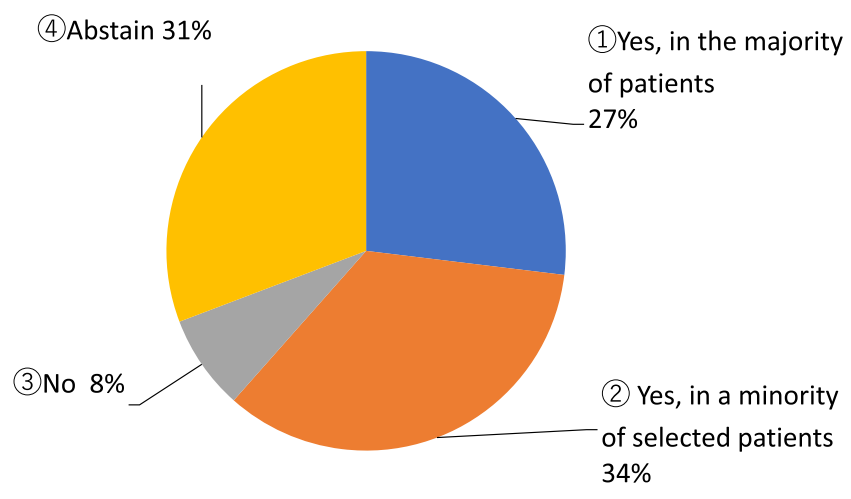

$\mathrm{CQ}=$ clinical question; $\mathrm{CSPC}=$ castration-sensitive prostate cancer.

Fig. 1 Do you prescribe osteoclast-targeting agents (zoledronic acid or denosumab) in patients with CSPC with bone metastases? [CQ6]

\section{Initial Diagnosis of Bone Metastases}

A meta-analysis of studies comparing fluoro-2deoxy-D-glucose positron emission tomography (PET), computed tomography (CT), magnetic resonance imaging (MRI), and bone scintigraphy for the diagnosis of bone metastases found that PET and MRI were comparable and that both were more accurate than CT and bone scintigraphy for the diagnosis of bone metastases [12]. On these questions, there was consensus within our panel, with $73 \%$ agreeing that bone scintigraphy alone is not sufficient for the initial diagnosis of bone metastases. There was also clear consensus, with the panel unanimously (100\%) agreeing that an MRI should be performed to confirm a diagnosis of metastases when there are discrepancies between the CT scan and bone scintigraphy.

\section{Management of Patients with CRPC}

The panel members were queried on their practices in treating patients with CRPC with different types of disease progression.

\section{The Reason for Stopping or Changing Treatment for CRPC}

There was strong consensus (91\%) that at least two of three criteria (prostate-specific antigen [PSA] progression, radiographic progression, and symptomatic progression) should be met before treatment was changed.

\section{Osteoclast-Targeted Agent for CRPC}

The panel was almost equally divided on the question of whether a change of drug or administration schedule is necessary if CRPC is diagnosed in a patient currently being treated with an osteoclast-targeting agent for CSPC with bone metastases. In contrast, for a patient who has not received such an agent, $83 \%$ recommended that an osteoclast-targeting agent be prescribed after the diagnosis of CRPC. The panel felt that the combined therapy would be an appropriate option, as suggested by the post hoc analysis from the COU-AA-302 study [13]. Further trials are necessary to confirm the effectiveness of this treatment strategy and optimal patient selection. 


\section{Treatment Strategies on Each Progression Status}

The panel members discussed treatment strategies for CRPC patients with multiple bone metastases but no visceral metastases (with good performance status [PS]).

Treatment strategy at diagnosis of CRPC (i.e., during PADT with good PS) Regarding treatment strategy at the diagnosis of CRPC, more than half of the panel members (62 vs. $50 \%)$ recommended a new androgen receptor (AR)targeted agent (ABI or ENZ) for those patients with rising PSA level and radiographic progression; for patients with symptomatic progression, however, the panel was more divided with regards to treatment, with $27 \%$ supporting treatment with ABI or ENZ ( \pm external beam radiotherapy [EBRT]), 36\% supporting treatment with DOC ( \pm EBRT), and $36 \%$ supporting treatment with Ra-223 ( \pm EBRT) (Fig. 2). There is as yet no clear evidence showing which CRPC patients, with bone metastases only, should be treated with the new AR-targeted agents DOC or Ra-223. Some panelists recommended that patients with aggressive bone disease should be treated with DOC, while other panelists commented that only a few patients will have aggressive disease. Some panelists suggested that Ra-223 is more likely to be used for symptomatic patients in the near future.

Treatment Strategy After Failure of ABI or ENZ, No Prior DOC, and with Good PS Regarding treatment strategy after the failure of treatment with $\mathrm{ABI}$ or ENZ or in case of primary resistance to $\mathrm{ABI}$ or $\mathrm{ENZ}, 60 \%$ of the panel members recommended DOC for patients with rising PSA. The opinions of the panel were more divided for cases of acquired resistance, with $38 \%$ supporting treatment with DOC, 29\% supporting treatment with Ra-223, and 19\% supporting treatment with another new ARtargeted agent. The panel was also split regarding the treatment of patients with radiographic progression, with $41 \%$ recommending DOC and $45 \%$ recommending Ra-223. For patients with symptomatic progression, 59\% recommended Ra-223 (Fig. 3).

The voting results suggest that a majority of the panel members believe that Ra-223 is an appropriate option for patients with symptomatic progression, although $60 \%$ of them at the time of the panel meeting did not have real-
For rising PSA (only)

【CQ28】
For radiographic progression (only bone metastases)【CQ29】
For symptomatic progression (only bone metastases)【CQ30】

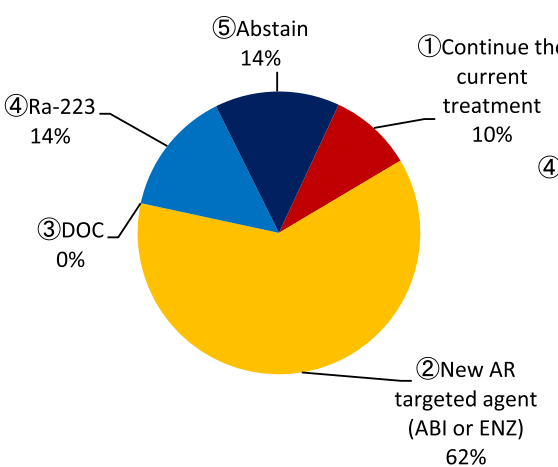

PSA

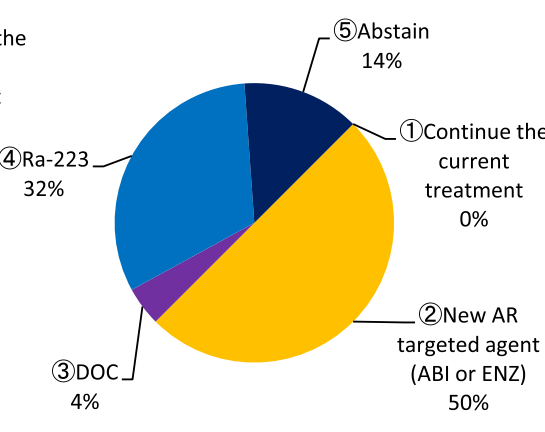

radiographic progression

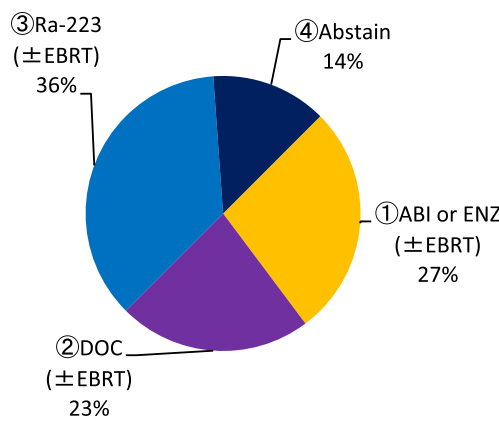

symptomatic progression

$\mathrm{ABI}=$ abiraterone; $\mathrm{AR}=$ androgen-receptor; $\mathrm{CQ}=$ clinical question; $\mathrm{CRPC}=$ castration-resistant prostate cancer; $\mathrm{EBRT}=\mathrm{external}$ beam radiotherapy; $E N Z=e n z a l u t a m i d e ; ~ P A D T=$ primary androgen deprivation therapy; $P S=$ performance status; $P S A=p r o s t a t e-s p e c i f i c$ antigen; Ra-223=radium-223 dichloride.

Fig. 2 Next preferred treatment in CRPC patients with multiple bone metastases (no visceral metastases) during PADT (with good PS). PSA Prostate-specific antigen 


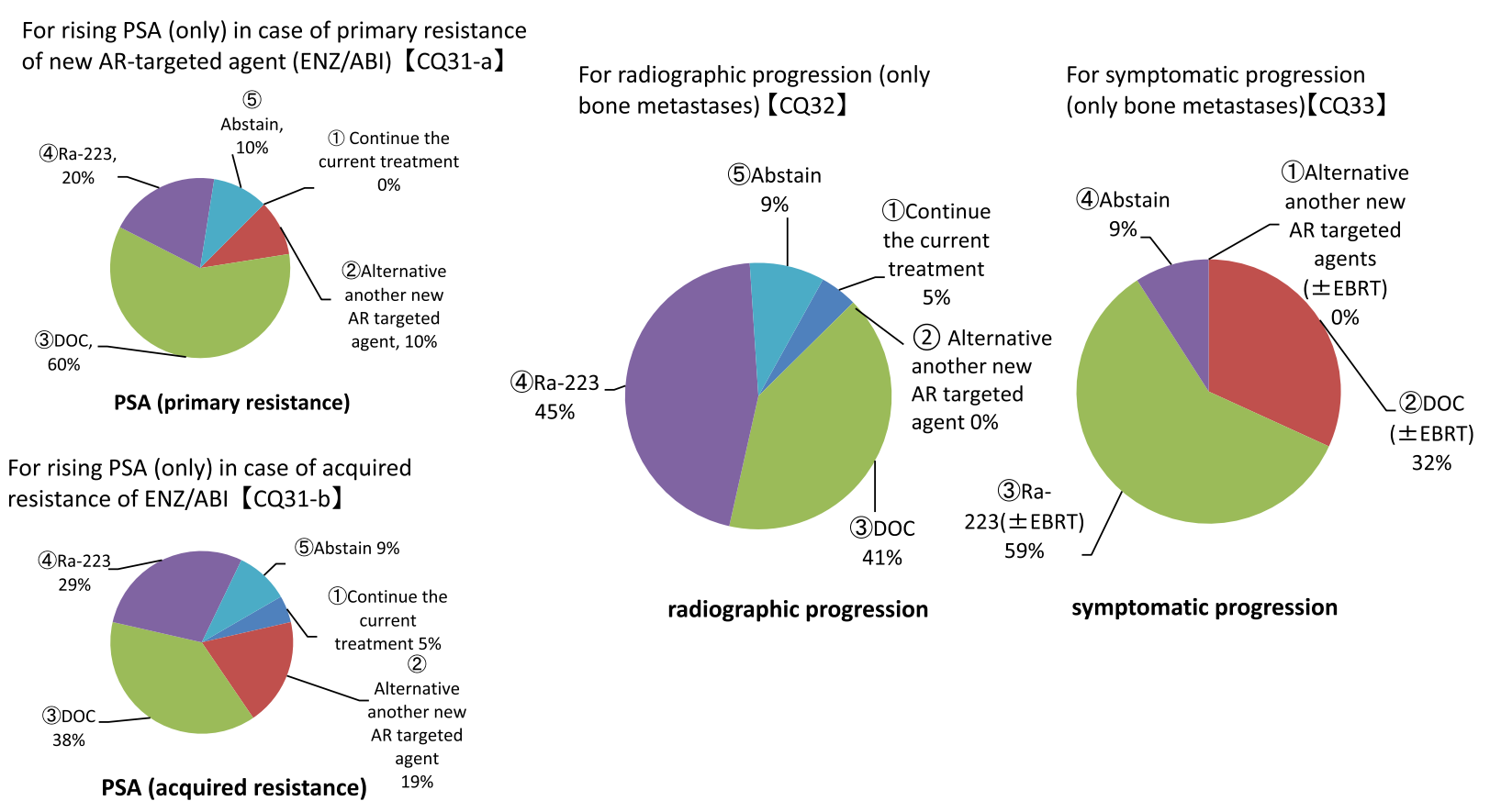

$A B I=$ abiraterone; $A R=$ androgen-receptor; $C Q=c l i n i c a l$ question; $C R P C=$ castration-resistant prostate cancer; $D O C=$ docetaxel; $E B R T=e x t e r n a l$

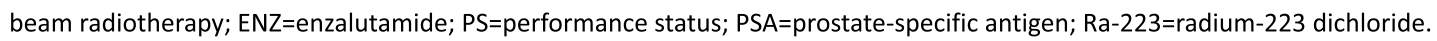

Fig. 3 Preferred next treatment in CRPC patients with multiple bone metastases (no visceral metastases) during treatment with new AR-targeted agent (ENZ/ABI), with no prior exposure to DOC and with good PS

world experience with the agent. When the panel members were asked whether they would want to prescribe Ra-223, in addition to new AR-targeted agents, if it were reimbursable, $67 \%$ voted 'Yes, in the majority of patients' and $14 \%$ voted 'Yes, in a minority of selected patients.' Further studies are necessary to develop evidence for reimbursement decisions.

\section{Treatment Strategy After DOC}

Regarding the next treatment option for patients who have received DOC for 10 cycles and whose PSA is stable and bone metastases on imaging indicate stable disease, $33 \%$ of the panel recommended discontinuation of DOC, while $22 \%$ recommended continuation of DOC and $22 \%$ recommended switching to Ra-223 (Fig. 4).

The panel members discussed whether the use of Ra-223 in this setting should be recommended or not. The voting results varied depending on the patient's status, with 50, 76, and $81 \%$ of the panel members recommending the use of Ra-223 in patients with a rising PSA only, with radiographic progression, and with symptomatic progression, respectively (Fig. 5).

\section{Treatment Strategy with Ra-223}

The panel members discussed the optimal timing of Ra-223 treatment. There was consensus that its use before chemotherapy was the more reasonable option rather than treatment after chemotherapy. The rationale for this recommendation is that a course of Ra-223 therapy requires a 6-month interval, and the time at which subsequent chemotherapy can be initiated requires careful evaluation.

Ra-223 is associated with hematological adverse events. As a result, the panel recommended performing regular blood tests at least every 2-4 weeks. 


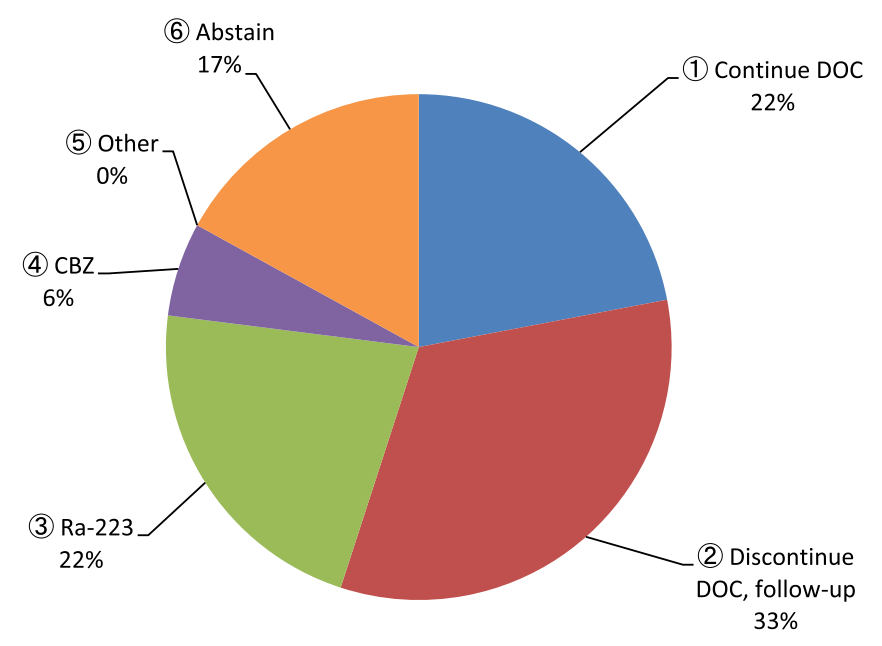

$\mathrm{CBZ}=$ cabazitaxel; $\mathrm{CQ}=$ clinical question; $\mathrm{CRPC}=$ castration-resistant prostate cancer; $\mathrm{DOC}=$ docetaxel; $\mathrm{PSA}=$ prostate-specific antigen.

Fig. 4 What is the next treatment option for CRPC patients with multiple bone metastases (no visceral metastasis) who have received DOC for 10 cycles and whose PSA is stable and bone metastases on imaging indicate stable disease? [CQ35]

Do you prescribe Ra-223 for rising PSA (only)? 【CQ36】

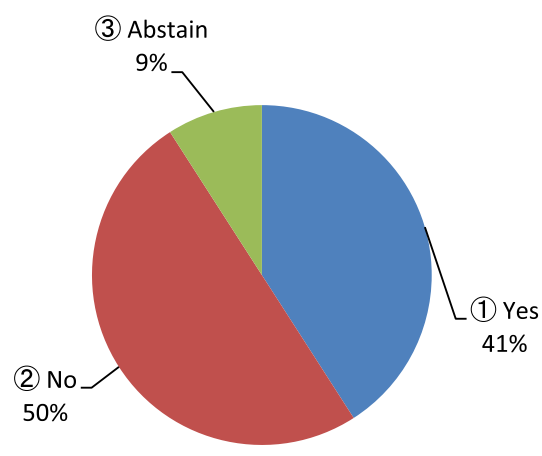

PSA
Do you prescribe Ra-223 for radiographic progression (only bone metastases)?【CQ37】

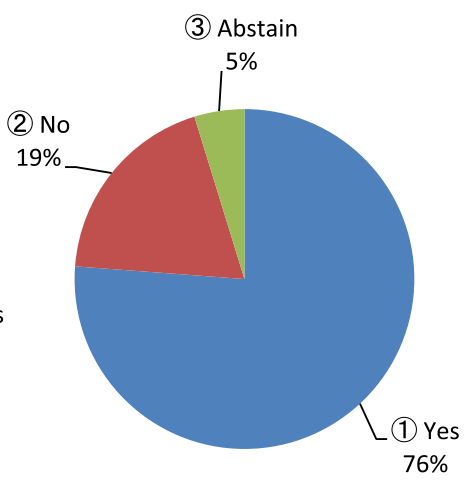

radiographic progression
Do you prescribe Ra-223 for symptomatic progression (only bone metastases)?[CQ38】

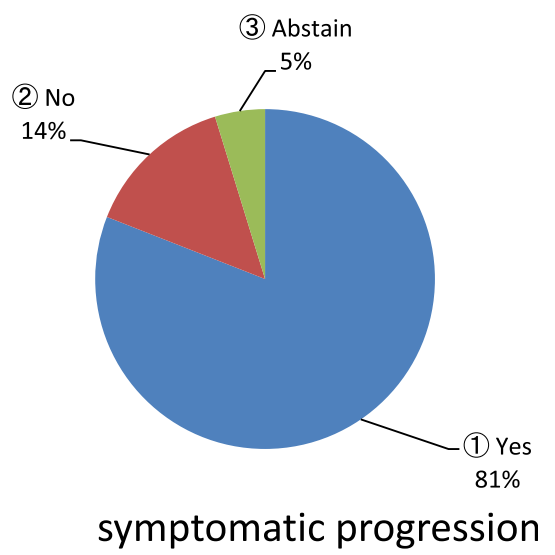

$\mathrm{CQ}=$ clinical question; $\mathrm{CRPC}=$ castration-resistant prostate cancer; $\mathrm{DOC}=$ docetaxel; $\mathrm{PS}=$ performance status; $\mathrm{PSA}=$ prostate-specific antigen; Ra-223=radium-223 dichloride.

Fig. 5 The use of Ra-223 in CRPC patients with multiple bone metastases (no visceral metastases) during DOC (with good PS) 


\section{Managing Bone Metastases in Patients with CRPC}

The panel members addressed many questions on their experiences and preferences for managing and treating bone metastases in patients with CRPC.

\section{Monitoring Bone Metastases}

Regarding treatment monitoring of patients with CRPC with bone metastases, $52 \%$ of the panel members recommended bone scintigraphy on a regular basis. The Prostate Cancer Working Group 3 (PCWG3) recommended a monitoring frequency of 8-9 weeks for the first 24 weeks, then every 12 weeks for bone scans as well as CT/MRI [14].

As to monitoring during treatment with Ra223, the panel strongly (91\%) recommended monitoring with CT scans to detect lymph node and/or visceral metastases. Altogether, $83 \%$ of the panel members also recommended regular monitoring of alkaline phosphatase (ALP) during treatment of CRPC with bone metastases.

\section{Definition of Progression of Bone Metastases}

To evaluate metastatic bone progression, the PCWG2 outlined the $2+2$ rule that recommends using two additional scans to confirm the appearance of at least two new lesions compared to an initial post-treatment scan. This method was maintained in PCWG3 and further extended to define the progression from nonmetastatic to metastatic CRPC [15]. The rule is intended to distinguish bone scan 'flare' from actual disease progression. However, implementation of this rule in real-world practice does appear to be haphazard, with fewer than half of panelists adopting this recommendation for assessing progression of bone metastases by imaging. Discussion showed that the PCWG3 recommendation was generally considered applicable for the clinical trial setting.

The panel reached a consensus (77\%) that at least two of three criteria (PSA progression, radiographic progression, and symptomatic progression) should be used as the definition of progression.

\section{Quantification of Bone Metastases}

The bone scan index (BSI), which is obtained using a computer-aided bone scan evaluation system, is anticipated to become an objective and quantitative clinical tool for evaluating bone metastases in prostate cancer [16]. Most panelists did not use BSI in clinical practice, and BSI was thought by some panelists to be premature for use in practice.

\section{Re-Biopsy/Biopsy in CRPC}

In 2014, a European Expert Consensus Panel emphasized the importance of biopsy to further understand the heterogeneity of prostate cancer and to move treatment planning toward a decision-making that was more individualized to the needs of the patient [17].

Conversely, in this forum, when asked if a rebiopsy of the primary tumor for patients who are diagnosed as CRPC is necessary, $77 \%$ of the panel voted 'No.' As to biopsy of the metastatic site, $64 \%$ voted 'No.' Most panelists considered re-biopsy of CRPC to have little significance for therapeutic decision-making and more significance for research.

\section{Response to Bone Pain}

The panel discussed the best treatment option for bone pain and recommended EBRT for patients who develop isolated pelvic bone pain and Ra-223 for patients who develop multiple or diffused bone pain at the pelvis and spine. Ra-223 following EBRT would be an appropriate option as well.

\section{DISCUSSION}

The main objective of this meeting was to share opinions among experts and to provide guidelines that would facilitate clinicians to successfully incorporate Ra-223 into CRPC treatment. At the time of the meeting, $60 \%$ of the panel members had no experience with Ra-223 treatment in actual practice and the optimal treatment strategy with Ra-223 had not yet been established. However, there was consensus for a number of key areas, including appropriate patient selection and optimal timing of treatment with Ra-233, for which the consensus was 
that patients with symptomatic metastatic CRPC and Ra-223 before chemotherapy, respectively, would benefit the most patients.

For patients with CSPC, CAB therapy was recommended by most panelists, although by contrast half of the panelists in the APCCC 2015 were opposed to $\mathrm{CAB}$. $\mathrm{CAB}$ has more efficacy than luteinizing hormone-releasing hormone (LHRH) agonists alone and can prevent flare phenomenon, but following the introduction of LHRH antagonists and new AR-targeted drugs in clinical practice, the significance of $\mathrm{CAB}$ as a treatment option is being re-considered.

New findings have been reported since the meeting and these should be taken into account when a treatment for metastatic CSPC is being considered. According to a systematic review and meta-analysis, DOC at the beginning of ADT therapy for metastatic CSPC resulted in significantly longer OS and PFS than that seen with ADT therapy alone [11]. At the time of the meeting in September 2016, this benefit had not been confirmed; $62 \%$ of the panel wanted to use DOC with ADT in patients with CSPC with bone metastases, and 23\% used this regimen in their practice. The panel stressed that DOC with ADT should be used only in appropriate patients and that careful patient selection is crucial. According to the randomized CHAARTED trial [18], the regimen was most effective in patients with higher volume disease; however, those patients with high-volume disease may have a higher risk of chemotherapy intolerance, and the definition of 'high tumor volume' has not been established. Therefore, it was also stressed by the panelists that other factors, including Gleason score, response to ADT, and patient characteristics, should be taken into consideration, and that new biomarkers must be explored.

The publication of the results of two important studies have resulted in changes in the treatment recommendations for metastatic CSPC since this consensus forum was held. The LATITUDE study showed that the addition of $\mathrm{ABI}$ and prednisone to ADT therapy to treat newly diagnosed, high-risk metastatic CSPC improved OS when compared to ADT therapy alone [19]. The STAMPEDE trial showed similar results, with improved OS and higher rates of failure free survival seen with the addition of
$\mathrm{ABI}$ and prednisone to $\mathrm{ADT}$ therapeutic regimen in a more heterogeneous patient population that included those with locally advanced or metastatic CSPC [20]. As a result of these studies, the addition of ABI and prednisone to ADT either before or after chemotherapy has been approved in Japan, as well as the USA and other countries, for high-risk CSPC [21].

Most panelists recommended that patients be monitored for osteoporosis and that treatment be provided if necessary during ADT. All panelists recommended using MRI when there are discrepancies between bone scintigram and CT findings. Almost all panelists recommended monitoring ALP during CRPC treatment.

Osteoclast-targeting agents were recommended for CRPC with bone metastases, and osteoclast-targeted agents can be used in combination with Ra-223. Most panelists recommended Ra-223 for patients with bone metastases only and with radiographic or symptomatic progression.

For other clinical questions, consensus between panelists was harder to determine and requires results from ongoing and future clinical studies and progress on regulatory issues. The panel was divided on the treatment strategy for patients with oligometastases, with half recommending drug treatment and others recommending additional therapies, including radical therapy and radiation. The diagnosis of oligometastases is important, and the significance of MRI or PET must be considered. There were different opinions on the definition of 'castration,' with regards to the threshold testosterone levels of $<50 \mathrm{ng} / \mathrm{dL}$ (conventional) or $<20 \mathrm{ng} / \mathrm{dL}$. In the COU-AA-02 study of ABI acetate, prognosis was different between threshold testosterone levels of $>20$ and $\leq 20 \mathrm{ng} / \mathrm{dL}$, but in practice almost all patients have $<20 \mathrm{ng}$ / dL testosterone with an LHRH agonist.

Regarding the evaluation of progression, fewer than half of the panelists recommended the use of PCWG3 or BSI criteria for monitoring. Many panelists thought that the PCWG3 criteria seem difficult and that the BSI criteria are too preliminary for use in clinical practice. The timing for changing treatment by scintigram extension of disease (EOD) grading was divided between EOD 1-2, 2-3, and 3-4 and is probably 
dependent on which treatment is considered: Ra-223, DOC, or a new AR-targeting treatment.

The treatment choice by panelists for patients with CRPC with bone metastases varied depending on whether the disease status was PSA-only, radiographic progression, or symptomatic progression. It was recommended that bone metastases with PSA-only progression be treated with a new AR-targeted agent and that symptomatic bone metastases be treated with Ra-223. The combination therapy of ABI and Ra223 was studied in a phase 3 randomized double-blind setting (ERA 223; NCT02043678) for CRPC patients with bone metastases, but due to the observation of an increased risk of fractures and death in the combination arm, the trial was unblinded in December 2017 before the scheduled end of the study. Analysis of these study data is currently being performed $[22,23]$.

The discussions and opinions of 27 Japanese experts on the treatment of prostate cancer are reported here. However, there are limitations to these data. First, these data represent the voting results of the meeting, and the treatment selections were not determined from the actual prescribing records. Due the progress of this therapeutic area, the participants may currently have different opinions.

This report is based on a consensus meeting held in 2016, and subsequent trial results and evidence influencing current therapeutic standards are not reflected in all of the voting results. However, it is important for physicians to know how our understanding of the optimal use of therapy has evolved to what is seen today by knowing the consensus formed at each milestone of development. Being familiar with these developmental steps is useful to improving our understanding of the rationale behind standard therapies and reinforces the education needed by physicians who must provide optimal treatments in this therapeutic area that has an increasing complexity of choices.

\section{CONCLUSIONS}

The panel was in consensus that the optimal timing of Ra-223 treatment is before chemotherapy rather after chemotherapy because a course of
Ra-223 therapy requires a 6-month interval, and the time to which subsequent chemotherapy administration can be initiated requires careful evaluation. Data from this meeting inform the development of future discussions or contributions to the development of new guidelines.

\section{ACKNOWLEDGEMENTS}

Funding. This study and the article processing charges were funded by Bayer Yakuhin Ltd., Tokyo, Japan.

Medical Writing and Other Editorial Assistance. The authors acknowledge medical writing support by Masami Kajiura and Aya Tokaji, MDS-CMG Inc., Tokyo, Japan, with funding from Bayer Yakuhin Ltd., Tokyo, Japan. Their services encompassed preparation of the first draft, preparing tables and figures, and incorporating authors' revisions, all under the direction of the authors. Bayer Yakuhin had the opportunity to review the manuscript as a funding sponsor, but did not have the authority to influence the content.

Authorship. All named authors meet the International Committee of Medical Journal Editors (ICMJE) criteria for authorship for this article, take responsibility for the integrity of the work as a whole and have given their approval for this version to be published.

Author Contributions. ST: conceptualization; methodology; steering committee member; drafting of clinical questions; original draft preparation. SK, NN, NS, KS, and HS: conceptualization; methodology; steering committee member; drafting of clinical questions. YK: supervision of the project; conceptualization; methodology; chairperson of the steering committee; drafting of clinical questions. All authors were present at the consensus meeting and voted on the clinical questions; they were all involved in the development and approved the final manuscript. The authors of this manuscript did not receive any honorarium for their writing and involvement in the developing of this 
manuscript. Funding for the expert panel meeting was provided by Bayer Yakuhin, at which the speakers for the meeting received a speakers' fee.

Disclosures. Shunji Takahashi reported receiving lecture fees from Eisai and DaiichiSankyo and research grants from AstraZeneca, Daiichi-Sankyo, Chugai Pharmaceutical, Novartis Pharma, Bayer, and Parexel International, and receiving meeting and medical writing financial support for this manuscript from Bayer Yakuhin Ltd., Tokyo, Japan. Norio Nonomura received consulting and meeting fees from Bayer. Nobuo Shinohara received research grants from Astellas and Ono Yakuhin and honoraria from GSK, Novartis, Pfizer, and Bayer, and also reports receiving meeting and medical writing financial support for this manuscript from Bayer Yakuhin Ltd., Tokyo, Japan. Kazuhiro Suzuki reported consulting and meeting expenses from Bayer, research grants from Astellas, Takeda, and Daiichi-Sankyo, and honoraria for speaking and educational writing from Astellas, Takeda, Daiichi-Sankyo, AstraZeneca, Janssen, Nihonkayaku, Bayer, and Fuji Film; he also reported receiving meeting and medical writing fincancial support for this manuscript from Bayer Yakuhin Ltd., Tokyo, Japan. Hiroyoshi Suzuki reported receiving honoraria and/or research grants from Astellas, Takeda, AstraZeneca, Bayer, Chugai, Novartis Pharma, Janssen Pharma, Nihon Kayaku, and Daiichi-Sankyo, and received an honorarium from Nihon Medi-Physics for serving as a medical specialist; he also reports receiving meeting and medical writing financial support for this manuscript from Bayer Yakuhin Ltd., Tokyo, Japan. Takefumi Satoh currently has another position in Sato Takefumi Zenritsusen Clinic, and reported receiving meeting and medical writing financial support for this manuscript from Bayer Yakuhin Ltd., Tokyo, Japan. Toshiyuki Yoneda reported receiving a consultant fee from Daiichi-Sankyo and receiving meeting and medical writing financial support for this manuscript from Bayer Yakuhin Ltd., Tokyo, Japan. Takao Kamai reported receiving honoraria from Astellas and Sanofi, and receiving meeting and medical writing financial support for this manuscript from Bayer Yakuhin Ltd., Tokyo, Japan. Takashi Mizowaki reported receiving a grant from Eisai, speaker fees from Astellas, Philips Electronics Japan, Takeda, Hitachi, Valiant Medical Systems, Brain Labo, Medicon, Electa, Asuka Pharma, Daiichi-Sankyo, and Bayer, and to receiving meeting expenses from MDS; he also reported receiving meeting and medical writing financial support for this manuscript from Bayer Yakuhin Ltd., Tokyo, Japan. Nobuaki Matsubara reported receiving speaking honoraria from Astellas, Astra Zeneca, Bayer, Eisai, Janssen, MSD, Novartis, Ono, Phizer, and Sanofi and to have received research funding from Novartis, Ono, and Sanofi; he has also received meeting and medical writing financial support for this manuscript from Bayer Yakuhin Ltd., Tokyo, Japan. Hiroji Uemura reported receiving consultation fees from Bayer and to receiving meeting and medical writing financial support for this manuscript from Bayer Yakuhin Ltd., Tokyo, Japan. Akira Yokomizo reported receiving speaking honoraria from Astellas, Takeda, Daiichi-Sankyo, Novartis Pharma, AstraZeneca, and Janssen and also to receiving meeting and medical writing financial support for this manuscript from Bayer Yakuhin Ltd., Tokyo, Japan. Mana Yoshimura reproted receiving research funding from Nihon Medi-Physics and also to have received meeting and medical writing financial support for this manuscript from Bayer Yakuhin Ltd., Tokyo, Japan. Seigo Kinuya, Nobuo Shinohara, Katsumasa Nakamura, Ukihide Tateishi, Hiroyuki Horikoshi, Tsukasa Igawa, Mitsuru Koizumi, Takeo Kosaka, Hideaki Miyake, Atsushi Mizokami, Takashi Mizowaki, Naoki Nakamura, Masahiro Nozawa, Takeo Takahashi, Motohide Uemura, and Yoshiyuki Kakehi have each reported that they have received meeting and medical writing financial support for this manuscript from Bayer Yakuhin Ltd., Tokyo, Japan.

Compliance with Ethics Guidelines. This article does not contain any studies with human participants or animals performed by any of the authors.

Data Availability. All data generated or analyzed during this study are included in this published article as supplementary information files. 
Open Access. This article is distributed under the terms of the Creative Commons Attribution-NonCommercial 4.0 International License (http://creativecommons.org/licenses/ by-nc/4.0/), which permits any noncommercial use, distribution, and reproduction in any medium, provided you give appropriate credit to the original author(s) and the source, provide a link to the Creative Commons license, and indicate if changes were made.

\section{REFERENCES}

1. National Cancer Center (Japan), Cancer Registration and Statistics 2017. http://ganjoho.jp/reg_stat/ statistics/stat/short_pred.html. Accessed 26 Mar 2017.

2. Parker C, Nilsson S, Heinrich D, et al. Alpha emitter radium-223 and survival in metastatic prostate cancer. N Engl J Med. 2013;369(3):213-23. https:// doi.org/10.1056/NEJMoa1213755.

3. Sartor O, Coleman R, Nilsson S, et al. Effect of radium-223 dichloride on symptomatic skeletal events in patients with castration-resistant prostate cancer and bone metastases: results from a phase 3, double-blind, randomised trial. Lancet Oncol. 2014;15(7):738-46. https://doi.org/10.1016/S14702045(14)70183-4.

4. Gillessen S, Omlin A, Attard G, et al. Management of patients with advanced prostate cancer: recommendations of the St Gallen Advanced Prostate Cancer Consensus Conference (APCCC) 2015. Ann Oncol. 2015;26(8):1589-604. https://doi.org/10. 1093/annonc/mdv257.

5. Hsu C-C, Sandford BA. The Delphi technique: making sense of consensus. Pract Assess Res Eval. 2007;12(10):1-8.

6. Japanese Urological Association. Clinical guideline for prostate cancer 2012. Bunkyo-ku, Tokyo: Kanehara-Shuppan; 2012. (in Japanese).

7. Thomas C, Bögemann M, König F, et al. Advanced Prostate Cancer Consensus Conference (APCCC) 2015 in St. Gallen: Critical review of the recommendations on diagnosis and therapy of metastatic prostate cancer by a German expert panel. Urologe A. 2016;55(6):772-82. https://doi.org/10.1007/ s00120-016-0030-8 (in German).

8. Ost P, Jereczek-Fossa BA, As NV, et al. Progressionfree survival following stereotactic body radiotherapy for oligometastatic prostate cancer treatment-naive recurrence: a multi-institutional analysis. Eur Urol. 2016;69(1):9-12. https://doi.org/ 10.1016/j.eururo.2015.07.004.

9. Azzam G, Lanciano R, Arrigo S, et al. SBRT: an opportunity to improve quality of life for oligometastatic prostate cancer. Front Oncol. 2015;5:101. https://doi.org/10.3389/fonc.2015.00101.

10. James ND, Sydes MR, Clarke NW, et al. Addition of docetaxel, zoledronic acid, or both to first-line long-term hormone therapy in prostate cancer (STAMPEDE): survival results from an adaptive, multiarm, multistage, platform randomised controlled trial. Lancet. 2016;387(10024):1163-77. https://doi.org/10.1016/S0140-6736(15)01037-5.

11. Vale CL, Burdett S, Rydzewska LH, et al. Addition of docetaxel or bisphosphonates to standard of care in men with localised or metastatic, hormone-sensitive prostate cancer: a systematic review and metaanalyses of aggregate data. Lancet Oncol. 2016;17(2):243-56. https://doi.org/10.1016/S14702045(15)00489-1.

12. Yang HL, Liu T, Wang XM, Xu Y, Deng SM. Diagnosis of bone metastases: a meta-analysis comparing ${ }^{18}$ FDG PET, CT, MRI and bone scintigraphy. Eur Radiol. 2011;21(12):2604-17. https://doi.org/10. 1007/s00330-011-2221-4.

13. Scher HI, Morris MJ, Stadler WM, et al. Trial design and objectives for castration-resistant prostate cancer: updated recommendations from the Prostate Cancer Clinical Trials Working Group 3. J Clin Oncol. 2016;34(12):1402-18. https://doi.org/10. 1200/JCO.2015.64.2702.

14. ASCO Annual Meeting, The Prostate Cancer Working Group 3 (PCWG3) consensus for trials in castration-resistant prostate cancer (CRPC). http://meet inglibrary.asco.org/content/149791-156. Accessed 30 Mar 2017.

15. Uemura K, Miyoshi Y, Kawahara T, et al. Prognostic value of a computer-aided diagnosis system involving bone scans among men treated with docetaxel for metastatic castration-resistant prostate cancer. BMC Cancer. 2016;16:109. https://doi. org/10.1186/s12885-016-2160-1.

16. Fitzpatrick JM, Bellmunt J, Fizazi K, et al. Optimal management of metastatic castration-resistant prostate cancer: highlights from a European Expert Consensus Panel. Eur J Cancer. 2014;50(9):1617-27. https://doi.org/10.1016/j.ejca. 2014.03.010.

17. Saad F, Shore N, Van Poppel H, et al. Impact of bone-targeted therapies in chemotherapy-naïve metastatic castration-resistant prostate cancer 
patients treated with abiraterone acetate: post hoc analysis of study COU-AA-302. Eur Urol. 2015;68(4):570-7. https://doi.org/10.1016/j.eururo. 2015.04.032.

18. Sweeney CJ, Chen YH, Carducci M, et al. Chemohormonal therapy in metastatic hormone-sensitive prostate cancer. N Engl J Med. 2015;373(8):737-46. https://doi.org/10.1056/NEJMoa1503747.

19. Fizazi K, Tran N, Fein L, LATITUDE Investigators, et al. Abiraterone plus prednisone in metastatic, castration-sensitive prostate cancer. N Engl J Med. 2017;377(4):352-60. https://doi.org/10.1056/ NEJMoa1704174.

20. James ND, de Bono JS, Spears MR, STAMPEDE Investigators, et al. Abiraterone for prostate cancer not previously treated with hormone therapy.
N Engl J Med. 2017;377(4):338-51. https://doi.org/ 10.1056/NEJMoa1702900.

21. Janssen Biotech, Inc. Zytiga(r) (Abiraterone acetate). Janssen Biotech, Inc. Horsham, PA. 2018. http:// www.janssenlabels.com/package-insert/productmonograph/prescribing-information/ZYTIGA-pi. pdf. Accessed 21 Nov 2018.

22. Parker C, Heidenreich A, Nilsson S, Shore N. Current approaches to incorporation of radium-223 in clinical practice. Prostate Cancer Prostatic Dis. 2018;21(1):37-47. https://doi.org/10.1038/s41391017-0020-y.

23. Sartor O, de Bono JS. Metastatic prostate cancer. N Engl J Med. 2018;378(7):645-57. https://doi.org/ $10.1056 /$ nejmra1701695. 